\title{
A CATEGORICAL INVARIANT FOR GEOMETRICALLY RATIONAL SURFACES WITH A CONIC BUNDLE STRUCTURE
}

\author{
MARCELLO BERNARDARA AND SARA DURIGHETTO
}

\begin{abstract}
We define a categorical birational invariant for minimal geometrically rational surfaces with a conic bundle structure over a perfect field via components of a natural semiorthogonal decomposition. Together with the similar known result on del Pezzo surfaces, this provide a categorical birational invariant for geometrically rational surfaces.
\end{abstract}

\section{INTRODUCTION}

In recent years the study of the derived category of an algebraic variety has been widely developed. It is clear now that semiorthogonal decompositions can provide a useful tool in order to detect the geometrical structure of a variety. In particular its interest focus in finding a birational invariant to be used, for example, to study the rationality of the variety.

In this context, the first author and M. Bolognesi [6] introduced the concept of categorical representability and formulated the following question: is a rational variety always categorically representable in codimension 2? Analogously, is it possible to characterize obstruction to rationality via natural components of some semiorthogonal decomposition which cannot be realized in codimension 2? On the complex field, for example, if we consider a $V_{14}$ Fano threefold $X$, its derived category admits a semiorthogonal decomposition with only one nontrivial component $\mathcal{A}_{X}$. For a smooth cubic threefold $Y$ we can also find a decomposition with only one nontrivial component $\mathcal{A}_{Y}$, and and Kuznetsov showed that $\mathcal{A}_{X}$ is equivalent to $\mathcal{A}_{Y}$ if $Y$ is the unique cubic threefold birational to $X$ [12]. This suggests that one could consider $\mathcal{A}_{X}$ as a birational invariant.

In the case of complex conic bundles $\pi: X \rightarrow S$ over a minimal rational surface the situation is quite well-known. A necessary condition to rationality of $X$ is that the intermediate Jacobian $J(X)$, as principally polarized Abelian variety, is the direct sum of the intermediate Jacobian of smooth projective curves. It follows for example that smooth cubic threefolds are not rational [7]. From a categorical point of view it is possible to characterize the rationality of the conic bundle from the semiorthogonal decomposition of the derived category. By Kuznetsov [11] we have

$$
\mathrm{D}^{\mathrm{b}}(X)=\left\langle\Phi \mathrm{D}^{\mathrm{b}}(D, \mathcal{B}), \pi^{*} \mathrm{D}^{\mathrm{b}}(S)\right\rangle,
$$

where $\mathcal{B}$ is the sheaf of even parts of Clifford algebras associated to the quadratic form defining the fibration and $\Phi: \mathrm{D}^{\mathrm{b}}(S, \mathcal{B}) \rightarrow \mathrm{D}^{\mathrm{b}}(S)$ is a fully faithful functor from the derived category of $\mathcal{B}$-algebras over $S$. If $S$ is rational the only nontrivial part for this semiorthogonal decomposition must then be contained in the component $\mathrm{D}^{\mathrm{b}}(S, \mathcal{B})$. If $S$ is minimal, the first author and Bolognesi proved that $X$ is rational if and only if $\mathrm{D}^{\mathrm{b}}(S, \mathcal{B})$ has a decomposition whose components are derived categories of smooth curves or exceptional objects [5]. 
All those results holds on the complex field $\mathbb{C}$, but we want to study the problem over an arbitrary perfect field $k$. Auel and the first author worked out the case of del Pezzo surfaces $[2]^{1}$. Given a minimal del Pezzo surface $S$ of degree $d$ and Picard rank 1, a natural subcategory $\mathcal{A}_{S} \subset \mathrm{D}^{\mathrm{b}}(S)$ is defined by the orthogonal complement to the structure sheaf. In [2], a category $\mathrm{GK}_{S}$ can be defined, roughly speaking, as the product of all components of $\mathcal{A}_{S}$ which are not representable in dimension 0 , and it is a birational invariant. Such Griffiths-Kuznetsov component, where it is defined, is then the suitable birational invariant to detect the rationality of the given variety. Our aim is to extend this approach to the other class of geometrically rational minimal surfaces, that is, conic bundles.

The precise definition of such an invariant is given in Definition 10. Roughly speaking, we define the Griffiths-Kuznetsov component to be the direct sum of subcategories of $\mathrm{D}^{\mathrm{b}}(S)$ which are not representable in dimension 0. However, unlikely in the case of del Pezzo surfaces, there is no, to the best of the authors' knowledge, argument to prove that the (natural) decomposition we choose to define $\mathrm{GK}_{S}$ is unique up to mutations. This motivates the involved case-by-case definition, and a fundamental part of this work is to prove that $\mathrm{GK}_{S}$ is indeed well-defined. Our main result is the following.

Theorem 1. Let $k$ be a perfect field and $S$ be a geometrically rational surface birational to a conic bundle over $k$. The Griffiths-Kuznetsov component $\mathrm{GK}_{S}$ is well defined and is a birational invariant.

Recall the classification of minimal geometrically rational surfaces over an arbitrary field (see, e.g., [9]): minimal conic bundles are one of the two possible classes of such surfaces, namely the ones with Picard rank two, the other being del Pezzo surfaces with Picard rank one. Combining Theorem 1 with the results from [2], we obtain the following result.

Theorem 2. Let $S$ be a geometrically rational surface over a perfect field $k$. Then the Griffiths-Kuznetsov component $\mathrm{GK}_{S}$ is well-defined and it is a birational invariant.

Notations. Functors of geometric origin between derived categories will be denoted underived (i.e. $f^{*}$ instead of $L f^{*}$ for the pull-back via a morphism). Given a $k$-algebra $A$, the notation $\mathrm{D}^{\mathrm{b}}(k, A)$ stands for the $k$-linear bounded derived category of coherent $A$-modules.

Acknowledgments. The authors are grateful to Stéphane Lamy for providing and discussing the example in remark 4, and to Jérémy Blanc and Michele Bolognesi for fruitful conversations.

\section{BASICS ON GEOMETRICALLY RATIONAL SURFACES}

In this section we will introduce some useful and known results. Let $k$ be a perfect field and $\bar{k}$ an algebraic closure. Let us consider $S$, a smooth projective geometrically integral surface over $k$. We say that $S$ is geometrically rational if $\bar{S}:=S \times_{k} \bar{k}$ is $\bar{k}$-rational. A field extension $l$ of $k$ is a splitting field for $S$ if $S \times_{k} l$ is birational to $\mathbb{P}_{l}^{2}$ through a sequence of monoidal transformations centered at closed $l$-points.

A smooth projective surface $S$ is minimal over $k$ if every birational morphism $\phi: S \rightarrow Y$, defined over $k$, to a smooth variety $Y$ is an isomorphism. If $k$ is algebraically closed, the

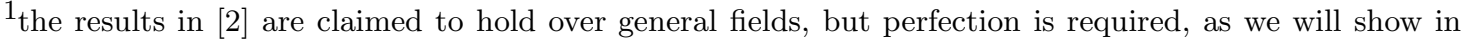
Remark 4, to ensure that evey birational map can be factored into Sarkisov links as in [10].
} 
only minimal rational surfaces are the projective plane and projective bundles over $\mathbb{P}^{1}$. Over a general field, we have the following classification (see, e.g., [9]).

Proposition 3. Let $S$ be a minimal geometrically rational surface over $k$. Then $S$ is one of the following:

(i) $S=\mathbb{P}_{k}^{2}$ is the projective plane, so $\operatorname{Pic}(S)=\mathbb{Z}$, generated by the hyperplane $\mathcal{O}(1)$;

(ii) $S \subset \mathbb{P}_{k}^{3}$ is a smooth quadric and $\operatorname{Pic}(S)=\mathbb{Z}$, generated by the hyperplane section $\mathcal{O}(1)$

(iii) $S$ is a del Pezzo surface with $\operatorname{Pic}(S)=\mathbb{Z}$, generated by the canonical class $\omega_{S}$;

(iv) $S$ is a conic bundle $f: S \rightarrow C$ over a geometrically rational curve, with $\operatorname{Pic}(S) \simeq$ $\mathbb{Z} \oplus \mathbb{Z}$.

2.1. Elementary links. We recall some elements of the Sarkisov program which describes the factorization of a birational map between minimal rational surfaces in elementary links [10]. Let $\pi: S \rightarrow Y$ be a minimal geometrically rational surface with an extremal contraction. Then either $Y$ is a point and $S$ is a minimal surface with Picard rank 1 or $Y$ is a curve and $S$ is a conic bundle with Picard rank 2. If $\pi: S \rightarrow Y$ and $\pi^{\prime}: S^{\prime} \rightarrow Y^{\prime}$ are two extremal contractions, an elementary link is a birational map $\phi: S \rightarrow S^{\prime}$ of one of the following types:

Type I) There is a commutative diagram

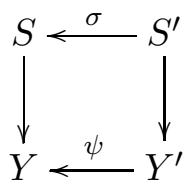

where $\phi=\sigma^{-1}, \sigma: S^{\prime} \rightarrow S$ is a Mori divisorial elementary contraction and $\psi$ : $Y^{\prime} \rightarrow Y$ is a morphism. In this case, $Y=\operatorname{Spec}(k), \rho(S)=1, S$ is a minimal del Pezzo, and $S^{\prime} \rightarrow Y^{\prime}$ is a conic bundle over a geometrically rational curve.

Type II) There is a commutative diagram

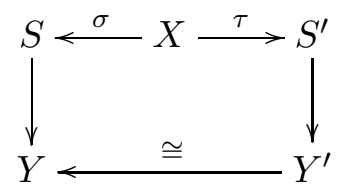

where $\phi=\tau \circ \sigma^{-1}, \sigma: X \rightarrow S$ and $\tau: X \rightarrow S^{\prime}$ are Mori divisorial elementary contractions. In this case, $S$ and $S^{\prime}$ have the same Picard number, and are hence either both del Pezzo surfaces (and $Y$ is a point) or both conic bundles (and $Y$ is a geometrically rational curve).

Type III) There is a commutative diagram

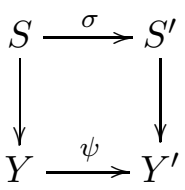

where $\phi=\sigma, \sigma: S \rightarrow S^{\prime}$ is a Mori divisorial elementary contraction and $\psi: Y \rightarrow Y^{\prime}$ is a morphism. Links of type III are nothing but inverse of links of type I. 
Type IV) There is a commutative diagram

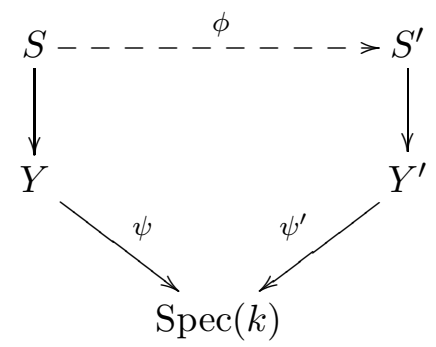

where $S \simeq S^{\prime}$ are isomorphic, $Y$ and $Y^{\prime}$ are geometrically rational curves and $\psi$ and $\psi^{\prime}$ are the structural morphisms. Then both $S$ and $S^{\prime}$ are conic bundles and the link amounts to a change of conic bundle structure on $S$.

Any birational map $\phi: S \rightarrow S^{\prime}$ between minimal geometrically rational surfaces can be factored through elementary links, and Iskovskikh gives the complete list of all possible such links [10]. We note that the Picard rank is invariant under links of type II and IV, while it changes under links of type I and III. Moreover, if we suppose that $S$ is not rational the list of links of type I (and hence of their inverses of type III) is very limited: either $S$ is of degree 8 with a point of degree 2 , and $S^{\prime}$ is of degree 6 and the curve $C$ can be rational (according to $S$ being a quadric or not), or $S$ is of degree 4 , has a rational point and $S^{\prime}$ is of degree 3 and $C$ is a rational curve.

Remark 4 . If $k$ is not perfect, than a birational map may not be decomposable in a finite sequence of elementary links centered at closed points. An example of such map was given in $[13, \mathrm{Rmk} .1 .3]$ : if $k=(\mathbb{Z} / 2 \mathbb{Z})[t]$, the birational map $\phi$ of $\mathbb{P}_{k}^{2}$ given by

$$
\left[x_{0}: x_{1}: x_{2}\right] \rightarrow\left[x_{0} x_{2}: x_{1} x_{2}: x_{0}^{2}+t x_{1}^{2}\right]
$$

has $[\sqrt{t}: 1: 0]$ as a base point, and such a point is never defined over a separable field extension of $k$.

\section{BASICS ON DERIVED CATEGORIES}

3.1. Categorical representability. Using semiorthogonal decompositions, one can define a notion of categorical representability for triangulated categories. In the case of smooth projective varieties, this is inspired by the classical notions of representability of cycles, see [6]. We refrain here to recall standard notions of semiorthogonal decompositions, exceptional objects, and mutations, the interested reader can refer to [1]. Let us just recall a nonstandard definition of exceptional object.

Definition 5. Let $A$ be a division (not necessarily central) simple $k$-algebra (i.e., the center of $A$ could be a field extension of $k$ ), and $\mathcal{A}$ a $k$-linear triangulated category. An object $V$ of $\mathcal{A}$ is called $A$-exceptional if

$$
\operatorname{Hom}(V, V)=A \text { and } \operatorname{Hom}(V, V[r])=0 \quad \text { for } \quad r \neq 0 .
$$

An exceptional object in the classical sense of the term [8, Def. 3.2] is a $k$-exceptional object. By exceptional object, we mean $A$-exceptional for some division $k$-algebra $A$.

Example 6. Let $A$ be a central simple algebra over $k$ and $X:=S B(A)$ the Severi-Brauer variety associated to it, and let $n=\operatorname{dim} X$. The Quillen vector bundle $V$ is a rank $n+1$ 
indecomposable vector bundle whose base change to a splitting field is $\mathcal{O}(1)^{\oplus n+1}$, and is in particular an $A$-exceptional object [14].

Definition 7. A triangulated category $\mathcal{A}$ is representable in dimension $m$ if it admits a semiorthogonal decomposition

$$
\mathcal{A}=\left\langle\mathcal{A}_{1}, \ldots, \mathcal{A}_{r}\right\rangle
$$

and for each $i=1, \ldots, r$ there exists a smooth projective $k$-variety $Y_{i}$ with $\operatorname{dim} Y_{i} \leq m$, such that $\mathcal{A}_{i}$ is equivalent to an admissible subcategory of $\mathrm{D}^{\mathrm{b}}\left(Y_{i}\right)$.

The motivation for the above definition is the possibility to formulate the following question:

Question 8. Let $X$ be a smooth projective $k$-variety of dimension $n$. Does $X$ rational imply $\mathrm{D}^{\mathrm{b}}(X)$ categorically representable in dimension $n-2$ ?

In this work, we consider the above question for surfaces, and we are hence interested in characterizing categories which are representable in dimension 0. This was done in [2].

Lemma 9. A triangulated category $\mathcal{A}$ is representable in dimension 0 if and only if there exists a semiorthogonal decomposition

$$
\mathcal{A}=\left\langle\mathcal{A}_{1}, \ldots, \mathcal{A}_{r}\right\rangle,
$$

such that for each $i$, there is a $k$-linear equivalence $\mathcal{A}_{i} \simeq \mathrm{D}^{\mathrm{b}}\left(K_{i} / k\right)$ for a separable field extension $K_{i} / k$

3.2. Conic bundles. We recall a natural semiorthogonal decomposition of the derived category of a surface with conic bundle structure, following the work of Kuznetsov [11] and its generalization to general fields [3]. Let $S$ be a surface over the field $k$ with a structure of conic bundle $\pi: S \rightarrow C$ over a geometrically rational curve. Such conic bundle is associated to a quadratic form $q: E \rightarrow L$ on a locally free $\mathcal{O}_{C}$-module $E$ of rank 3 . Denote by $\mathcal{O}_{S / C}(1)$ the restriction to $S$ of the line bundle $\mathcal{O}_{\mathbb{P} E / C}(1)$, and let $\mathcal{B}$ be the even Clifford algebra associated to the form $q$, which is a locally free $\mathcal{O}_{C}$-algebra whose isomorphism class is invariant for $\pi: S \rightarrow C$.

Under these conditions, we have that $\pi^{*}: \mathrm{D}^{\mathrm{b}}(C) \rightarrow \mathrm{D}^{\mathrm{b}}(S)$ is fully faithful, and there exist a fully faithful functor $\Phi: \mathrm{D}^{\mathrm{b}}(C, \mathcal{B}) \rightarrow \mathrm{D}^{\mathrm{b}}(S)$ such that

$$
\mathrm{D}^{\mathrm{b}}(S)=\left\langle\pi^{*} \mathrm{D}^{\mathrm{b}}(C), \Phi \mathrm{D}^{\mathrm{b}}(C, \mathcal{B})\right\rangle .
$$

Moreover, since $C$ is a geometrically rational curve, there is a simple $k$-algebra $A$ (trivial if and only if $C \simeq \mathbb{P}_{k}^{1}$ ) such that $C=S B(A)$. In particular, there is an $A$-exceptional object $V$, which is either $\mathcal{O}(1)$ if $C \simeq \mathbb{P}_{k}^{1}$ or the Quillen bundle $V$ as in example 6 if $C$ is not rational, such that

$$
\mathrm{D}^{\mathrm{b}}(C)=\left\langle\mathcal{O}_{C}, V\right\rangle=\left\langle V^{*}, \mathcal{O}_{C}\right\rangle
$$

It follows that we can refine the semiorthogonal decomposition of $S$ (abusing of notation, setting $\left.V:=\pi^{*} V\right)$ as follows:

$$
\mathrm{D}^{\mathrm{b}}(S)=\left\langle\mathcal{O}_{S}, V, \Phi \mathrm{D}^{\mathrm{b}}(S, \mathcal{B})\right\rangle .
$$

Now, let $\bar{\pi}: \bar{S} \rightarrow \mathbb{P}_{\bar{k}}^{1}$ be the base change of the conic bundle to the algebraic closure. Such a conic bundle is not necessarily a Hirzebruch surface and can indeed be not minimal, and have a finite number, say $r$, of singular fibers which are given by two lines meeting in 
a point. We can pick one line in each fiber and denote such set of lines by $F_{1}, \ldots, F_{r}$. The Picard rank of $\bar{S}$ is then $2+r$, and there is a semiorthogonal decomposition obtained by considering the $\bar{k}$-minimal model $\bar{S} \rightarrow S_{0}$, which is a Hirzebruch surface:

$$
\mathrm{D}^{\mathrm{b}}(\bar{S})=\left\langle\mathcal{O}_{\bar{S}}, \mathcal{O}_{\bar{S}}(F), \mathcal{O}_{\bar{S}}(\Sigma), \mathcal{O}_{\bar{S}}(\Sigma+F), \mathcal{O}_{F_{1}}, \ldots, \mathcal{O}_{F_{r}}\right\rangle,
$$

where $F$ is the general fiber of $\bar{\pi}, \Sigma$ is a section of $\bar{\pi}$.

We finally notice that the base change of the semiorthogonal decomposition (3.1) is exactly the semiorthogonal decomposition (3.2): indeed, either $C$ is rational and we already have $V=\mathcal{O}_{S}(F)$, or $C$ is not rational, $V$ has rank 2 and we have $\bar{V}=\mathcal{O}_{\bar{S}}(F)^{\oplus 2}$. The latter generates the same category as $\mathcal{O}_{\bar{S}}(F)$ since we are considering thick subcategories.

It follows that the base change of $\Phi \mathrm{D}^{\mathrm{b}}(C, \mathcal{B})$ to $\bar{S}$ is the subcategory

$$
\left\langle\mathcal{O}_{\bar{S}}, \mathcal{O}_{\bar{S}}(F)\right\rangle^{\perp}=\left\langle\mathcal{O}_{\bar{S}}(\Sigma), \mathcal{O}_{\bar{S}}(\Sigma+F), \mathcal{O}_{F_{1}}, \ldots, \mathcal{O}_{F_{r}}\right\rangle
$$

\section{Links of type I/III And the Definition of the Griffiths-KuZnetsov COMPONENT}

We are going to construct a birational invariant for geometrically rational surfaces with a conic bundle $\pi: S \rightarrow C$ as the collection of subcategories in the semiorthogonal decomposition (3.1) which are not representable in dimension 0. Such an invariant will match the one constructed in [2] in the case where $S$ is birational to a minimal del Pezzo surface. Hence, we first have to deal with links of type I/III in the non-rational cases to give a proper definition. Indeed, the subcategory $\Phi \mathrm{D}^{\mathrm{b}}(C, \mathcal{B})$ can admit semiorthogonal decompositions and even exceptional object if $S$ is birational to a quadric or to a del Pezzo surface of degree 4. These cases were already treated in [2], and we quickly recall them.

Let $S^{\prime}$ be a minimal non-rational del Pezzo surface of degree 8 with a point of degree 2. Then, $S^{\prime}$ is an involution surface in a Severi-Brauer threefold $S B(B)$, and there is an associated even Clifford algebra $\mathcal{C}$, which is a simple algebra whose center is a degree two field extension of $k$, and a semiorthogonal decomposition

$$
\mathrm{D}^{\mathrm{b}}\left(S^{\prime}\right)=\left\langle\mathrm{D}^{\mathrm{b}}(k), \mathrm{D}^{\mathrm{b}}(k, B), \mathrm{D}^{\mathrm{b}}(k, \mathcal{C})\right\rangle,
$$

where the first category is generated by $\mathcal{O}_{S^{\prime}}$ and the second one either by $\mathcal{O}_{S^{\prime}}(1)$ (in which case $B=0$ and $S^{\prime}$ is a quadric) or by the restriction of the Quillen bundle of $S B(B)$ to $S^{\prime}$ (in the case where $S^{\prime}$ is not a quadric). It follows that the Griffiths-Kuznetsov component for $S^{\prime}$ should be $\mathrm{GK}_{S^{\prime}}:=\mathrm{D}^{\mathrm{b}}(k, \mathcal{C})$ if $S^{\prime}$ is a quadric and $\mathrm{GK}_{S^{\prime}}:=\mathrm{D}^{\mathrm{b}}(k, \mathcal{C}) \oplus \mathrm{D}^{\mathrm{b}}(k, B)$ if $S^{\prime}$ is not a quadric. In this case, such a category is not shown to be a birational invariant in [2], and this is due to the existence of a link of type I, from which follows that the birational class of $S^{\prime}$ contains minimal conic bundles of degree 6 . Indeed, the blow-up of a degree 2 point $S \rightarrow S^{\prime}$ is a conic bundle $\pi: S \rightarrow C$, with $C$ either rational if $S^{\prime}$ is a quadric or non-rational if $S^{\prime}$ is not a quadric.

In $[2, \S \mathrm{B}]$, it is proved that the component we want to construct is indeed related to the standard semiorthogonal decomposition of the conic bundle as follows: writing $C=S B(A)$ we have that $A$ and $B$ are Morita-equivalent (note that $B$ has order dividing 2 since it has an involution defining $S^{\prime}$ ), and that there is a degree 2 extension $l / k$ and a semiorthogonal decomposition:

$$
\mathrm{D}^{\mathrm{b}}(C, \mathcal{B})=\left\langle\mathrm{D}^{\mathrm{b}}(l), \mathrm{D}^{\mathrm{b}}(k, \mathcal{C})\right\rangle
$$


It follows that the components which are (potentially) not representable in dimension 0 in the standard decomposition (3.1) are exactly the ones we considered above for $S^{\prime}$.

Let $S^{\prime}$ be a minimal del Pezzo surface of degree 4 with a rational point. Note that $S^{\prime}$ is not rational. In particular, there is semiorthogonal decomposition

$$
\mathrm{D}^{\mathrm{b}}\left(S^{\prime}\right)=\left\langle\mathcal{O}_{S^{\prime}}, \mathcal{A}_{S^{\prime}}\right\rangle
$$

and $\mathrm{GK}_{S^{\prime}}:=\mathcal{A}_{S^{\prime}}$ is expected to be the good candidate for the birational invariant we are looking for. This case neither was treated in [2], since, again, the existence of a link of type I implies that the birational class of $S^{\prime}$ contains minimal conic bundles of degree 3 . Indeed, the blow-up of a rational point $S \rightarrow S^{\prime}$ is a conic bundle $\pi: S \rightarrow \mathbb{P}^{1}$.

In [2, A.2] (see also [3]), it is proved that the component we want to construct is indeed related to the standard semiorthogonal decomposition of the conic bundle since there is an equivalence $\mathrm{D}^{\mathrm{b}}(C, \mathcal{B}) \simeq \mathcal{A}_{S^{\prime}}$. It follows that the component which is (potentially) not representable in dimension 0 in the standard decomposition (3.1) is exactly the one we considered above for $S^{\prime}$.

Now that we have analyzed all the possible links of type I/III between non-rational surfaces, we can give the definition of the Griffiths-Kuznetsov component of a conic bundle.

Definition 10. Let $S$ be a surface with a structure of conic bundle $\pi: S \rightarrow C$ over a geometrically rational smooth curve $C$. If $S$ is minimal, the Griffiths-Kuznetsov component $\mathrm{GK}_{S}$ of $S$ is defined as follows:

(i) if $S$ is rational, $\mathrm{GK}_{S}=0$;

(ii) if $S=C_{1} \times C_{2}$ where $C_{i}$ is a geometrically rational curve with associated Azumaya algebra $A_{i}$, then $\mathrm{GK}_{S}$ is the sum of those between $\mathrm{D}^{\mathrm{b}}\left(k, A_{1}\right), \mathrm{D}^{\mathrm{b}}\left(k, A_{2}\right)$ and $\mathrm{D}^{\mathrm{b}}\left(k, A_{1} \otimes A_{2}\right)$ which are not equivalent to $\mathrm{D}^{\mathrm{b}}(k)$ (equivalently, the algebra is not Brauer-trivial);

(iii) if $C \simeq \mathbb{P}^{1}$ and $S$ is birational to a non-rational quadric with associated even Clifford algebra $\mathcal{C}$, then $\mathrm{GK}_{S}=\mathrm{D}^{\mathrm{b}}(k, \mathcal{C})$

(iv) if $C \simeq \mathbb{P}^{1}$, and $S$ is neither rational nor birational to a quadric, $\mathrm{GK}_{S}=\mathrm{D}^{\mathrm{b}}\left(\mathbb{P}^{1}, \mathcal{B}\right)$;

(v) if $C$ is not rational with associated Azumaya algebra $A$, and $S$ is birational to a quadric with associated even Clifford algebra $\mathcal{C}$, then $\mathrm{GK}_{S}=\mathrm{D}^{\mathrm{b}}(k, A) \oplus \mathrm{D}^{\mathrm{b}}(k, \mathcal{C})$;

(vi) if $C$ is not rational with associated Azumaya algebra $A$, and $S$ is not birational to a quadric, then $\mathrm{GK}_{S}=\mathrm{D}^{\mathrm{b}}(k, A) \oplus \mathrm{D}^{\mathrm{b}}(C, \mathcal{B})$;

If $S$ is not minimal, the Griffiths-Kuznetsov component is $\mathrm{GK}_{S}=\mathrm{GK}_{S_{0}}$ for a minimal model $S \rightarrow S_{0}$.

Note that the term component is slightly abused here, since there are cases where $\mathrm{GK}_{S}$ is not a component of $\mathrm{D}^{\mathrm{b}}(S)$ but rather the direct sum of some components. Since we can operate mutations on semiorthogonal decompositions (and we will indeed do to proof the main theorem), we cannot in general give any canonical gluing of components contributing to $\mathrm{GK}_{S}$.

The rest of the paper is dedicated to the proof of Theorem 1. Note that we can restrict to minimal models. If $\pi: S \rightarrow C$ and $\pi^{\prime}: S^{\prime} \rightarrow C^{\prime}$ be are minimal conic bundle structures and $\phi: S \rightarrow S^{\prime}$ is a birational morphism, then $\phi$ can be decomposed in a finite number of links. The invariance of $\mathrm{GK}_{S}$ under links of type I and III has been studied above and 
follows by results from [2]. To complete the proof, we will prove the invariance under links of type II (Theorem 11) and type IV (Corollary 14).

\section{LINKS OF TYPE II}

Links of type II between conic bundles are the most common birational transformation, that is given by an elementary transformation along a closed fiber of the conic bundle structure. Let $\pi: S \rightarrow C$ be a conic bundle. To define a link of type II, pick a closed point $x \in S$ of degree $d$, and denote by $S_{x}$ the fiber of $\pi$ containing it. Then perform the blow-up of $x$ followed by the subsequent contraction of the fiber $S_{x}$. This gives a conic bundle $\pi^{\prime}: S^{\prime} \rightarrow C$ and a commutative diagram:

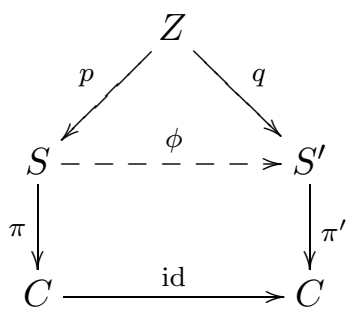

Theorem 11. In the above setting, we have $\mathrm{GK}_{S} \simeq \mathrm{GK}_{S^{\prime}}$.

Proof. Let $E$ be the exceptional divisor of $p$ and $E^{\prime}$ the exceptional of $q$. We denote by $f$ and $f^{\prime}$ the fibers of $\pi$ and $\pi^{\prime}$ in $Z$ respectively. Recall [10] that

$$
\begin{aligned}
p^{*}\left(-K_{X}\right) & =q^{*}\left(-K_{Y}\right)+d f-2 E^{\prime} \\
f & =f^{\prime} \\
E & =d f^{\prime}-E^{\prime}=p^{*}\left(-K_{X}\right)-q^{*}\left(-K_{Y}\right)+E^{\prime}
\end{aligned}
$$

Let $V$ be the Quillen bundle on $C$. Since the isomorphism class of $C$ is preserved under this link, so is the algebra $A$, and therefore the category $\mathrm{D}^{\mathrm{b}}(k, A)$.

We are left to prove that the equivalence class of the category $\mathcal{A}_{S}:=\mathrm{D}^{\mathrm{b}}(C, \mathcal{B})$ is also preserved. Over the algebraic closure, the category $\mathcal{A}_{\bar{S}} \subset \mathrm{D}^{\mathrm{b}}(\bar{S})$ admits the following semiorthogonal decomposition:

$$
\mathcal{A}_{\bar{S}}=\left\langle\mathcal{O}_{\bar{S}}(\Sigma), \mathcal{O}_{\bar{S}}(\Sigma+F), \mathcal{O}_{F_{1}}, \ldots, \mathcal{O}_{F_{r}}\right\rangle,
$$

where $F_{i}$ are given a choice of a line in each singular fibers of the fibration $\bar{S} \rightarrow \mathbb{P} \frac{1}{k}$, and $F$ and $\Sigma$ are respectively the fiber and the section of the conic bundle. Similarly, we have a semiorthogonal decomposition

$$
\mathcal{A}_{\overline{S^{\prime}}}=\left\langle\mathcal{O}_{\overline{S^{\prime}}}\left(\Sigma^{\prime}\right), \mathcal{O}_{\overline{S^{\prime}}}\left(\Sigma^{\prime}+F^{\prime}\right), \mathcal{O}_{F_{1}^{\prime}}, \ldots, \mathcal{O}_{F_{r}^{\prime}}\right\rangle .
$$

Over $\bar{k}$, we have that $\bar{S}$ is the blow up of $r$ points on a Hirzebruch surface $\mathbb{F}_{n}$, for some $n$, so that $K_{\bar{S}}=2 \Sigma+(n+2) F$. Similarly, $\overline{S^{\prime}}$ is the blow-up of $r$ points on a Hirzebruch surface $\mathbb{F}_{m}$ and one can see that the map $\bar{\phi}$ is obtained by lifting to $\bar{S}$ the composition of $d$ elementary transformations on $\mathbb{F}_{n}$ along fibers that do not contain the points blown-up by $\bar{S} \rightarrow \mathbb{F}_{n}$. In particular, $m=n-d$ and $K_{\overline{S^{\prime}}}=2 \Sigma^{\prime}+(n-d+2) F^{\prime}-E^{\prime}$. It follows that in our transformation: $\Sigma=\Sigma^{\prime}-E^{\prime}$.

Then we have the following equivalence of subcategories of $\mathrm{D}^{\mathrm{b}}(\bar{Z})$ :

$$
p^{*} \mathcal{A}_{\bar{S}} \otimes \mathcal{O}\left(E^{\prime}\right)=q^{*} \mathcal{A}_{\overline{S^{\prime}}} .
$$


Indeed, first note the singular fibers are preserved under the birational transformation $\phi$, we can choose $F_{i}^{\prime}$ such that $p^{*} F_{i}=q^{*} F_{i}^{\prime}$ for $i=1, \ldots, r$. Moreover $\mathcal{O}_{q^{*}} F_{i}$ does not change under tensor with $\mathcal{O}\left(E^{\prime}\right)$, since the exceptional divisor is not supported on singular fibers. Secondly, using the above relation $\Sigma=\Sigma^{\prime}-E^{\prime}$, it is not difficult to see that

$$
\langle\mathcal{O}(\Sigma), \mathcal{O}(\Sigma+F)\rangle \otimes \mathcal{O}\left(E^{\prime}\right)=\left\langle\mathcal{O}\left(\Sigma^{\prime}\right), \mathcal{O}\left(\Sigma^{\prime}+F^{\prime}\right)\right\rangle
$$

We can now conclude since the autoequivalence $\otimes\left(E^{\prime}\right)$ descends to an autoequivalence of $\mathrm{D}^{\mathrm{b}}(Z)$, since $E^{\prime}$ is defined over $k$. It follows, that $\mathcal{A}_{S}$ is equivalent to $\mathcal{A}_{S^{\prime}}$ and the proof is complete.

\section{LINKS OF TYPE IV}

A link of type IV is a birational self-transformation of a minimal surface $S$ exchanging two conic bundle structures $\pi_{i}: S \rightarrow C_{i}$, for $i=1,2$. We will than denote by $\mathcal{A}_{i}:=$ $\Phi_{i} \mathrm{D}^{\mathrm{b}}\left(C_{i}, \mathcal{B}_{i}\right) \subset \mathrm{D}^{\mathrm{b}}(S)$ to keep simple notations. In particular, the birational map $\phi: S \rightarrow$ $S$ fits a commutative diagram

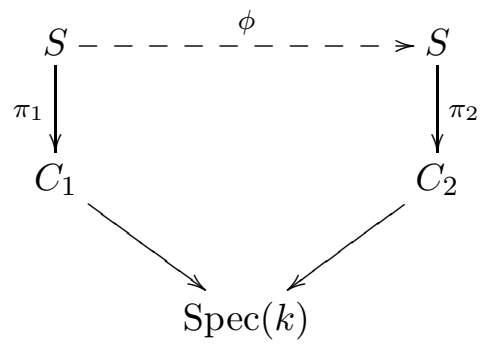

As proved in [10], $S$ must have degree $8,4,2$ or 1 , and the list of birational maps is quite limited. In this case, we need to prove that the Griffiths-Kuznetsov component is well defined, namely that it does not depend on the choice of semiorthogonal decomposition given by the different conic bundle structures. We proceed by a case by case analysis.

Proposition 12. Let $S$ be a degree 8 surface and $\phi$ a link of type $I V$. Then $\mathrm{GK}_{S}$ is well defined.

Proof. In this case, $S=C_{1} \times C_{2}$. The fact that $\mathrm{GK}_{S}$ is well-defined is proved in $[2, \S \mathrm{C}]$

Proposition 13. Let $S$ be a surface of degree 4, 2, or 1 , and $\phi$ a link of type IV. Then $\mathrm{GK}_{S}$ is well defined.

Proof. For $i=1,2$, consider the semiorthogonal decomposition

$$
\mathrm{D}^{\mathrm{b}}(S)=\left\langle\mathcal{O}_{S}, V_{i}, \mathcal{A}_{i}\right\rangle,
$$

and recall that $\left\langle\overline{V_{i}}\right\rangle=\left\langle\mathcal{O}_{\bar{S}}\left(F_{i}\right)\right\rangle$, for $F_{i}$ a geometric fiber of $\pi_{i}$, since either $C_{i}$ is rational and $V_{i}$ is such a line bundle, or $C_{i}$ is not rational and $\overline{V_{i}}=\mathcal{O}_{\bar{S}}\left(F_{i}\right)^{\oplus 2}$. Now we proceed by case by case analysis following the possibilities given by [10].

Degree 4. Assume $S$ has degree 4. We have $F_{1}=-K_{S}-F_{2}$, so that $V_{1}=V_{2}^{*} \otimes \omega^{*}$. It follows that $\left\langle V_{1}\right\rangle \simeq\left\langle V_{2}^{*}\right\rangle \simeq\left\langle V_{2}\right\rangle$, since $A_{2}^{o p}$ and $A_{2}$ are Brauer equivalent.

Now consider

$$
\mathrm{D}^{\mathrm{b}}(S)=\left\langle V_{2}^{*}, \mathcal{O}_{S}, \mathcal{A}_{2}\right\rangle=\left\langle V_{1}, \omega_{S}^{*}, \mathcal{A}_{2} \otimes \omega_{S}^{*}\right\rangle=\left\langle\mathcal{O}_{S}, \mathcal{A}_{2}, V_{1}\right\rangle
$$


where the first equality is given by the autoequivalence $\otimes \omega_{S}$ on $\mathrm{D}^{\mathrm{b}}(S)$ and the second is the mutation of $\left\langle\omega_{S}^{*}, \mathcal{A}_{2}^{\prime}\right\rangle$ to the left with respect to $V_{1}$. We can then mutate $\mathcal{A}_{2}$ to the right with respect to $V_{1}$ and obtain then $\mathcal{A}_{1}$, so that we have shown that $\mathcal{A}_{1} \simeq \mathcal{A}_{2}$ and we finished the proof.

Degree 2. Assume $S$ has degree 2. We have $F_{1}=-2 K_{S}-F_{2}$, so that $V_{1}=V_{2}^{*} \otimes\left(\omega^{*}\right)^{\otimes 2}$. It follows that $\left\langle V_{1}\right\rangle \simeq\left\langle V_{2}^{*}\right\rangle \simeq\left\langle V_{2}\right\rangle$, since $A_{2}^{o p}$ and $A_{2}$ are Brauer equivalent.

Now consider the first conic bundle structure and the semiorthogonal decompositions

$$
\mathrm{D}^{\mathrm{b}}(S)=\left\langle\mathcal{O}_{S}, V_{1}, \mathcal{A}_{1}\right\rangle=\left\langle V_{1}, \mathcal{A}_{1}, \omega_{S}^{*}\right\rangle=\left\langle\mathcal{A}_{1}^{\prime}, V_{1}, \omega_{S}^{*}\right\rangle
$$

where the first equality is the mutation of $\mathcal{O}_{S}$ to the right with respect to its orthogonal complement and the second one is the mutation of $\mathcal{A}_{1}$ to the left with respect to $V_{1}$, so that $\mathcal{A}_{1}^{\prime}={ }^{\perp}\left\langle V_{1}, \omega_{S}^{*}\right\rangle$ is equivalent to $\mathcal{A}_{1}$.

Now consider the second conic bundle structure and the semiorthogonal decompositions

$$
\begin{aligned}
\mathrm{D}^{\mathrm{b}}(S) & =\left\langle V_{2}^{*}, \mathcal{O}_{S}, \mathcal{A}_{2}\right\rangle=\left\langle V_{1},\left(\omega_{S}^{*}\right)^{\otimes 2}, \mathcal{A}_{2} \otimes\left(\omega_{S}^{*}\right)^{\otimes 2}\right\rangle= \\
& =\left\langle\omega_{S}^{*}, \mathcal{A}_{2} \otimes\left(\omega_{S}^{*}\right), V_{1}\right\rangle=\left\langle\mathcal{A}_{2}^{\prime}, \omega_{S}^{*}, V_{1}\right\rangle,
\end{aligned}
$$

where we first tensor with $\left(\omega_{S}^{*}\right)^{\otimes 2}$, then mutate $\left\langle\left(\omega_{S}^{*}\right)^{\otimes 2}, \mathcal{A}_{2} \otimes\left(\omega_{S}^{*}\right)^{\otimes 2}\right\rangle$ to the left with respect to its orthogonal complement, then mutate $\mathcal{A}_{2} \otimes \omega_{S}^{*}$ to the left with respect to $\omega_{S}^{*}$. It follows in particular that $\mathcal{A}_{2}^{\prime}={ }^{\perp}\left\langle\omega_{S}^{*}, V_{1}\right\rangle$ is equivalent to $\mathcal{A}_{2}$.

Finally, the two semiorthogonal decompositions give the full orthogonality between $V_{1}$ and $\omega_{S}^{*}$, so that $\left\langle\omega_{S}^{*}, V_{1}\right\rangle=\left\langle V_{1}, \omega_{S}^{*}\right\rangle$. This implies that $\mathrm{A}_{1}^{\prime}=\mathrm{A}_{2}^{\prime}$ and the proof follows.

Degree 1. Assume $S$ has degree 1. We have then that $C_{i}$ are rational and $F_{1}=$ $-4 K_{S}-F_{2}$. In particular, we only need to prove that the categories $\mathcal{A}_{1}$ and $\mathcal{A}_{2}$ are equivalent.

Let us consider the first conic bundle structure and the semiorthogonal decompositions:

$$
\begin{gathered}
\mathrm{D}^{\mathrm{b}}(S)=\left\langle\mathcal{O}\left(-F_{1}\right), \mathcal{O}_{S}, \mathcal{A}_{1}\right\rangle=\left\langle\mathcal{O}\left(F_{2}\right),\left(\omega_{S}^{*}\right)^{\otimes 4}, \mathcal{A}_{1} \otimes\left(\omega_{S}^{*}\right)^{\otimes 4}\right\rangle= \\
\quad=\left\langle\left(\omega_{S}^{*}\right)^{\otimes 3}, \mathcal{A}_{1} \otimes\left(\omega_{S}^{*}\right)^{\otimes 3}, \mathcal{O}\left(F_{2}\right)\right\rangle=\left\langle\left(\omega_{S}^{*}\right)^{\otimes 3}, \mathcal{O}\left(F_{2}\right), \mathcal{A}_{1}^{\prime}\right\rangle,
\end{gathered}
$$

where we first tensor by $\left(\omega_{S}^{*}\right)^{\otimes 4}$, then mutate $\left\langle\left(\omega_{S}^{*}\right)^{\otimes 4}, \mathcal{A}_{1} \otimes\left(\omega_{S}^{*}\right)^{\otimes 4}\right\rangle$ to the left with respect to its orthogonal complement, then mutate $\mathcal{A}_{1} \otimes\left(\omega_{S}^{*}\right)^{\otimes 3}$ to the right with respect to $\mathcal{O}\left(F_{2}\right)$.

Now we need to mutate $\mathcal{O}\left(F_{2}\right)$ to the left with respect to $\left(\omega_{S}^{*}\right)^{\otimes 3}$. To this end, let us calculate:

$$
\operatorname{Hom}^{i}\left(\left(\omega_{S}^{*}\right)^{\otimes 3}, \mathcal{O}\left(F_{2}\right)=H^{i}\left(S, \mathcal{O}\left(3 K_{S}+F_{2}\right)\right) .\right.
$$

First of all, note that $\left(3 K_{S}+F_{2}\right) \cdot F_{2}<0$, which implies that $H^{0}\left(S, \mathcal{O}\left(3 K_{S}+F_{2}\right)\right)=0$. Similarly, by Serre duality we have that $H^{2}\left(S, \mathcal{O}\left(3 K_{S}+F_{2}\right)\right)=H^{0}\left(S, \mathcal{O}\left(2 K_{S}+F_{1}\right)\right)=0$ since $\left(2 K_{S}+F_{1}\right) \cdot F_{1}<0$.

Finally we are left with $\operatorname{dim} H^{1}\left(S, \mathcal{O}\left(3 K_{S}+F_{2}\right)\right)=-\chi\left(\mathcal{O}_{S}, \mathcal{O}\left(3 K_{S}+F_{2}\right)\right)$. The latter can be calculated by Riemann-Roch:

$$
\chi\left(\mathcal{O}_{S}, \mathcal{O}\left(3 K_{S}+F_{2}\right)\right)=\frac{1}{2}\left(3 K_{S}+F_{2}\right) \cdot\left(2 K_{S}+F_{2}\right)+1=-1,
$$

since $K_{S} \cdot F_{2}=-2$ and $S$ has degree 1 . It follows, that there is a unique extension

$$
0 \longrightarrow \mathcal{O}\left(F_{2}\right) \longrightarrow \mathcal{F} \longrightarrow\left(\omega_{S}^{*}\right)^{\otimes 3} \longrightarrow 0,
$$


which has rank 2 and first Chern class $F_{2}-3 K_{S}$. Moreover, $\mathcal{F}$ is the result of the mutation of $\mathcal{O}\left(F_{2}\right)$ to the left with respect to $\left(\omega_{S}^{*}\right)^{\otimes 3}$, so that we end up with the decomposition

$$
\mathrm{D}^{\mathrm{b}}(S)=\left\langle\mathcal{F},\left(\omega_{S}^{*}\right)^{\otimes 3}, \mathcal{A}_{1}^{\prime}\right\rangle .
$$

Now consider the second conic bundle structure and the semiorthogonal decompositions:

$$
\mathrm{D}^{\mathrm{b}}(S)=\left\langle\mathcal{O}_{S}, \mathcal{O}\left(F_{2}\right), \mathcal{A}_{2}\right\rangle=\left\langle\mathcal{O}\left(F_{2}\right), \mathcal{A}_{2}, \omega_{S}^{*}\right\rangle=\left\langle\mathcal{O}\left(F_{2}\right), \omega_{S}^{*}, \mathcal{A}_{2}^{\prime}\right\rangle,
$$

where the first equality is the mutation of $\mathcal{O}_{S}$ to the right with respect to its right orthogonal, and $\mathcal{A}_{2}^{\prime}$ is the mutation of $\mathcal{A}_{2}$ to the left with respect to $\omega_{S}^{*}$ and is therefore equivalent to $\mathcal{A}_{2}$.

We mutate now $\mathcal{O}\left(F_{2}\right)$ to the right with respect to $\omega_{S}^{*}$. A calculation similar to the above one shows that there is exactly one nontrivial extension

$$
0 \longrightarrow \omega_{S}^{*} \longrightarrow \mathcal{G} \longrightarrow \mathcal{O}\left(F_{2}\right) \longrightarrow 0
$$

which has rank 2 and first Chern class $F_{2}-K_{S}$. Moreover, $\mathcal{G}$ is the result of the mutation of $\mathcal{O}\left(F_{2}\right)$ to the right with respect to $\omega_{S}^{*}$, and is an exceptional object. Thanks to Gorodentsev [8], exceptional bundles on $S$ are characterized by their rank and their first Chern class. Note that the $\mathcal{F}$ and $\mathcal{G}$ have both rank 2, while the first Chern class of $\mathcal{G}$ is the first Chern class of $\mathcal{F} \otimes \omega_{S}$. It follows that $\mathcal{G} \simeq \mathcal{F} \otimes \omega_{S}$ is the mutation of $\mathcal{O}\left(F_{2}\right)$ to the right with respect to $\omega_{S}^{*}$. We hence end up with the decompositions

$$
\begin{gathered}
\mathrm{D}^{\mathrm{b}}(S)=\left\langle\omega_{S}^{*}, \mathcal{F} \otimes \omega_{S}, \mathcal{A}_{2}^{\prime}\right\rangle=\left\langle\left(\omega_{S}^{*}\right)^{\otimes 2}, \mathcal{F}, \mathcal{A}_{2}^{\prime} \otimes \omega_{S}^{*}\right\rangle= \\
=\left\langle\mathcal{F}, \mathcal{A}_{2}^{\prime} \otimes \omega_{S}^{*},\left(\omega_{S}^{*}\right)^{\otimes 3}\right\rangle=\left\langle\mathcal{F},\left(\omega_{S}^{*}\right)^{\otimes 3}, \mathcal{A}_{2}^{\prime \prime}\right\rangle,
\end{gathered}
$$

where first we tensor by $\omega_{S}^{*}$, then mutate $\left(\omega_{S}^{*}\right)^{\otimes 2}$ to the right with respect to its right orthogonal, and $\mathcal{A}_{2}^{\prime \prime}$ is the left mutation of $\mathcal{A}_{2}^{\prime} \otimes\left(\omega_{S}^{*}\right)^{\otimes 2}$ to the right with respect to $\left(\omega_{S}^{*}\right)^{\otimes 3}$ and is therefore equivalent to $\mathcal{A}_{2}$. The proof follows then by comparison with 6.1.

Corollary 14. The Griffiths-Kuznetsov component is well-defined for minimal conic bundles and hence birational invariant under links of type $I V$.

\section{REFERENCES}

[1] A. Auel, and M. Bernardara, Cycles, derived categories, and rationality, in Surveys on Recent Developments in Algebraic Geometry, Proceedings of Symposia in Pure Mathematics 95, 199-266 (2017).

[2] A. Auel and M. Bernardara, Semiorthogonal decompositions and birational geometry of del Pezzo surfaces over arbitrary fields, Proc. London Math. Soc. (3) 117 (2018) 1-64.

[3] A. Auel, M. Bernardara, and M. Bolognesi, Fibrations in complete intersections of quadrics, Clifford algebras, derived categories, and rationality problems, Journal de Math. Pures et Appliques 102, 249-291 (2014)

[4] M. Bernardara, A semiorthogonal decomposition for Brauer-Severi schemes. Math. Nachr. 282 (2009), no. 10.

[5] M. Bernardara and M. Bolognesi, Derived categories and rationality of conic bundles, Compositio Math. 149 (2013), no. 11, 1789-1817.

[6] M. Bernardara and M. Bolognesi, Categorical representability and intermediate Jacobians of Fano threefolds. EMS Ser. Congr. Rep., Eur. Math. Soc., 2013.

[7] C. Clemens and P. Griffiths, The intermediate Jacobian of the cubic threefold. Ann. Math. 95 (1972), $281-356$.

[8] A.L. Gorodentsev, Exceptional bundles on surfaces with a moving anticanonical class, Izv. Akad. Nauk SSSR Ser. Mat. 52 (1988), no. 4, 740-757, 895; translation in Math. USSR-Izv. 33 (1989), no. 1, 67-83. 
[9] B. Hassett, Rational surfaces over nonclosed fields, in Arithmetic geometry 155-209, Clay Math. Proc., 8, Amer. Math. Soc., Providence, RI, 2009.

[10] V.A. Iskovskikh, Factorization of birational mappings of rational surfaces from the point of view of Mori theory (Russian) Uspekhi Mat. Nauk 51 (1996), no. 4(310), 3-72; translation in Russian Math. Surveys 51 (1996), no. 4, 585-652.

[11] A. Kuznetsov, Derived categories of quadric fibrations and intersections of quadrics, Adv. Math. 218 (2008), no. 5, 1340-1369.

[12] A. Kuznetsov, Derived category of cubic and $V_{14}$ threefold, Proc. V.A. Steklov Inst. Math. 246 (2004), 183-207.

[13] S. Lamy and S. Zimmermann, Signature morphisms from the Cremona group over a non-closed field, to appear in Journal of the European Mathematical Society.

[14] D. Quillen, Higher algebraic K-theory. I, Algebraic K-theory I: Higher $K$-theories (Proc. Conf., Battelle Memorial Inst., Seattle, Wash., 1972), Lecture Notes in Math., vol. 341, Springer, Berlin, 1973, pp. 85147.

Institut de Mathématiques de Toulouse, Université Paul Sabatier, 118 route de Narbonne, 31062 Toulouse Cedex 9, France

E-mail address: marcello.bernardara@math.univ-toulouse.fr

Institut de Mathématiques de Toulouse, Université Paul Sabatier, 118 route de Narbonne, 31062 Toulouse Cedex 9, France

E-mail address: drgsra@unife.it 